\title{
LA COMPRENSIÓN LECTORA Y SU RELACIÓN CON EL USO DE ESTRATEGIAS METACOGNITIVAS DE LECTURA EN UNIVERSITARIOS
}

\section{READING COMPREHENSION AND ITS RELATIONSHIP WITHTHE USE OF METACOGNITIVE READING STRATEGIES IN UNIVERSITY STUDENTS}

\author{
Marycarmen Chablé Tolosa \\ Instituto Valle Dorado, México \\ 1170544@alumno.um.edu.mx \\ https://orcid.org/0000-0003-4174-3356
}

\begin{abstract}
RESUMEN
La presente investigación, descriptiva y correlacional, tuvo como objetivo determinar si existe relación entre la comprensión lectora y el uso de estrategias metacognitivas de lectura en un grupo de 90 estudiantes de una universidad privada en el noreste de México. Según los resultados, existe relación significativa entre la comprensión lectora y las estrategias metacognitivas de lectura en universitarios, siendo el mejor modelo predictor de la comprensión lectora el conformado por las estrategias de apoyo y las de resolución de problemas. Se observó también que existe correlación negativa entre la comprensión lectora y la dimensión estrategias de apoyo de lectura. En cuanto a la dimensión estrategias de resolución de problemas, se encontró que fueron las estrategias más usadas por los estudiantes, mientras que las menos utilizadas fueron las estrategias de apoyo. Además, los hombres obtuvieron una mejor correlación entre la comprensión lectora y las estrategias metacognitivas de lectura que las mujeres.
\end{abstract}

Palabras clave: comprensión lectora, estrategias metacognitivas, estudiantes universitarios

\section{ABSTRACT}

This descriptive and correlational research aimed to determine if there is a relationship between reading comprehension and the use of metacognitive reading strategies in a group of 90 students from a private university in northeastern Mexico. According to the results, there is a significant relationship between reading comprehension and metacognitive reading strategies in university students, the best predictor model of reading comprehension being the one formed by support strategies and problem-solving strategies. It was also observed that there is a negative correlation between reading comprehension and the reading support strategies dimension. Regarding the problem-solving strategies dimension, it was found that these were the most used by the students, while the least used were the support strategies. 


\section{CHABLÉ TOLOSA}

Furthermore, men obtained a better correlation between reading comprehension and metacognitive reading strategies than women.

Keywords: reading comprehension, metacognitive strategies, university students

\section{Introducción}

De acuerdo con los resultados de la evaluación PISA 2015, aplicada a estudiantes de nivel secundario, la educación mexicana se mantiene en los últimos lugares de calidad educativa desde hace 15 años. En México, el 48\% de los alumnos evaluados registra conocimientos insuficientes en el área de ciencias, $42 \%$ en lectura y $57 \%$ en matemáticas, según el Instituto Nacional para la Evaluación de la Educación (INEE, 2016).

Es imprescindible para la educación que las nuevas generaciones aprendan a desarrollar habilidades y estrategias eficaces, ya que con ellas se pueden alcanzar niveles óptimos de aprendizaje autónomo. Esto permitirá que los alumnos mejoren su comprensión lectora y por consiguiente alcancen mejores resultados en las demás asignaturas (Salas Navarro, 2012).

Sin embargo, es alto el porcentaje de alumnos con deficiencias en comprensión lectora observado en las aulas de clase. Al respecto, diversas investigaciones han demostrado que hay maneras de mejorarla si se conocen sus elementos, se comprenden sus procesos y si se enseña correctamente a los alumnos cómo abordar los textos (Gómez López y Silas Casillas, 2012).

\section{Comprensión lectora}

La comprensión lectora desempeña una función central en la educación actual. En el proceso de enseñanza aprendizaje, que abarca desde la educación primaria hasta la universitaria, es nece- sario leer para apoderarse de diferentes conocimientos y su importancia radica en la cantidad, estilo y propósitos de la lectura. La comprensión lectora implica la habilidad de comprender, interpretar y dar sentido a diversos tipos de textos al relacionarlos con diferentes contextos (Salas Navarro, 2012).

La competencia lectora es una de las herramientas cognitivas más relevantes en el proceso de enseñanza-aprendizaje, pues permite entender el significado de la palabra, la construcción de oraciones y la emisión de ideas propias del lector. Su carácter transversal conlleva efectos positivos o negativos sobre el resto de las áreas académicas, lo cual significa que las dificultades del lector en comprensión de textos se transfieren al resto de las áreas curriculares. Resulta indispensable que el alumno domine esta competencia, que a la larga se reflejará en el aprendizaje y rendimiento académico (Beltrán Campos y Repetto Talavera, 2006; Omar y Bidin, 2015).

Leer es algo más que decodificar palabras y establecer significados. Involucra los cincos sentidos y permite al lector distinguir un panorama más amplio. Alaís Grillo et al. (2015) señalan que un lector es competente cuando puede comprender los diferentes niveles de un texto y desarrollar una actitud activa y participativa en su proceso lector.

\section{Niveles de comprensión lectora}

Considerando la comprensión como un proceso de interacción entre el texto 
y el lector, varios autores reconocen tres niveles de comprensión: literal, inferencial y crítico o evaluativo. En el nivel de comprensión literal, se estimula preferentemente a los sentidos y el lector reconoce las frases y las palabras clave del texto y capta lo que el texto dice sin una intervención muy activa de la estructura cognoscitiva e intelectual. Es como una reconstrucción del texto (Gordillo Alfonso y Flórez, 2009; Sánchez Carlessi, 2013).

En el nivel inferencial, el lector escudriña y establece asociaciones de significados que le permiten leer entre líneas, reconocer e inferir lo implícito; es decir, busca relaciones que van más allá de lo leído. Explica el texto más ampliamente y agrega informaciones y experiencias que le permiten formular nuevas ideas. Su meta es la elaboración de conclusiones (Basaraba et al., 2013; Green y Roth, 2013).

En el nivel crítico o evaluativo, de acuerdo con Oseda Gago y Cabezudo Ríos (2014), el lector es capaz de proyectar sus propias ideas sobre el texto leído. Este nivel se considera el ideal, ya que tiene un carácter evaluativo, que permite al lector apropiarse del contenido del texto, tomar una posición al respecto y elaborar su punto de vista (Díaz Pertuz y Quiroz Mosquera, 2016).

\section{Estrategias metacognitivas de lectura}

Aşıkcan y Saban (2018) definen las estrategias metacognitivas de lectura como el monitoreo consciente del proceso lector, en donde el sujeto interviene y evalúa cuando lo considera necesario, con la finalidad de mejorar la comprensión lectora. Además, pueden facilitar más el aprendizaje y el desempeño desarrollado, especialmente entre los estudiantes que se esfuerzan mucho por entender el con- texto escrito (Ahmadi et al., 2013).

Las estrategias metacognitivas pueden clasificarse en función del momento de uso: (a) antes de iniciar la lectura, para facilitar la activación de conocimientos previos, detectar y anticipar el contenido del texto y planificar el proceso lector, (b) durante la lectura, para facilitar al lector el reconocimiento de las distintas estructuras textuales, construir una representación mental del texto escrito y supervisar el proceso lector y (c) después de la lectura, para facilitar al lector el control del nivel de comprensión alcanzando, corregir errores de comprensión, elaborar una representación general y propia del texto escrito para efectuar procesos de transferencia del conocimiento obtenido mediante la lectura (Channa et al., 2015; Khoshsima y Samani, 2015; Schmitt y Baumann, 1990).

Las estrategias metacognitivas difieren de las cognitivas en que abarcan múltiples áreas. Mientras que es probable que las estrategias cognitivas se encapsulen dentro de un área temática, los lectores que aplican estrategias metacognitivas saben qué hacer cuando se enfrentan con dificultades en el aprendizaje y qué estrategias utilizar (Ahmadi et al., 2013).

La mayoría de las investigaciones (Dabarera et al., 2014; Habibian, 2015; Jou, 2015; Lee, 2018; Magogwe, 2013; Muñoz-Muñoz y Ocaña de Castro, 2017; Nasab y Motlagh, 2015; Smith-Keita, 2018) coinciden en que existe una estrecha y significativa correlación entre la comprensión lectora y el uso de estrategias metacognitivas de lectura. Este hallazgo representa un importante antecedente para abordar la presente investigación que tiene como finalidad promover el desarrollo de estrategias 
metacognitivas en la lectura de los estudiantes y así lograr mejores resultados en comprensión lectora, que repercuten en el logro académico.

Este estudio pretende contribuir con la búsqueda de información que permita conocer la importancia de crear un ambiente óptimo con estrategias novedosas para la comprensión lectora y que el alumno la valore como herramienta de estudio, no como un proceso aburrido y repetitivo. Con ello, se busca mejorar y fortalecer el aprendizaje de la lectura comprensiva, ya que uno de los problemas que más preocupa a los profesores de cualquier nivel escolar es la comprensión lectora de los estudiantes, dado que representa una de las habilidades cognitivas más importantes del ser humano y que lo acompaña durante toda su existencia.

\section{Metodología \\ Tipo de investigación}

El presente estudio tiene un enfoque cuantitativo con diseño descriptivo-correlacional.

\section{Participantes}

En el estudio participaron 90 estudiantes de cuarto año de diversas facultades de una universidad privada del noreste de México, de los cuales 24 eran estudiantes de la Facultad de Teología, 14 de la Facultad de Ingeniería y Tecnología, cinco de la Facultad de Administración, siete de la Facultad de Educación, siete de la Licenciatura en Terapia Física, nueve de la Licenciatura en Químico Clínico Biólogo, nueve de la Escuela de Artes y Comunicación y 15 de la Escuela de Ciencias Estomatológicas, cuyas edades oscilan entre los 20 y 35 años, matriculados en el ciclo escolar 2018-2019.

\section{Instrumentos}

Comprensión lectora. Para medir la comprensión lectora se utilizó la sección de comprensión de textos, del área de razonamiento verbal, de la Guía de ejercicios de la Prueba de Admisión a Estudios de Posgrado (PAEP), diseñada por el Instituto Tecnológico y de Estudios Superiores de Monterrey (2003). Esta sección evalúa la habilidad para analizar información escrita; contiene dos tipos de análisis: (a) el análisis tradicional, que presenta un pasaje seguido por preguntas sobre la idea principal, inferencias, conclusiones y vocabulario, y (b) el análisis crítico, que cuenta con dos pasajes, seguidos por preguntas donde el candidato debe interpretar, sintetizar, analizar y evaluar los elementos de los textos. Para determinar la consistencia del instrumento en la población del estudio, se administró una prueba piloto a un grupo con características similares a las de los participantes del estudio y se obtuvo un coeficiente de confiabilidad alfa de Cronbach de .651.

Estrategias metacognitivas de lectura. Para medir las estrategias metacognitivas de lectura, se utilizó el Inventario de Conciencia Metacognitiva de Estrategias Lectoras (MARSI), que incluye tres dimensiones y consta de 30 ítems evaluados en una escala Likert de cinco puntos: nunca o casi nunca hago eso (1) hasta siempre o casi siempre hago eso (5). La primera dimensión, de estrategias globales de lectura, cuenta con 13 ítems; la segunda, de estrategias de resolución de problemas, consta de ocho ítems, y la tercera dimensión, de estrategias de apoyo a la lectura, cuenta con nueve ítems.

La versión en español fue validada por Vallejos Atalaya (2012), con 827 estudiantes universitarios peruanos. Para 
determinar la consistencia del instrumento, se administró la prueba piloto a un grupo con características similares al del estudio, en donde el coeficiente alfa de Cronbach mostró un índice de .820 en el primer factor, un índice de confiabilidad alfa de Cronbach de .811 en el segundo factor y un índice de confiabilidad alfa de Cronbach de .753 en el tercer factor.

\section{Análisis de datos}

Se utilizó el coeficiente de correlación $r$ de Pearson para determinar la relación entre las estrategias metacognitivas de escritura y la comprensión lectora en los universitarios. Para comprobar si las estrategias metacognitivas de lectura eran predictoras significativas de la comprensión lectora en los estudiantes, se recurrió al análisis de regresión lineal múltiple.

\section{Resultados \\ Análisis descriptivo}

En esta sección se describen las medias aritméticas de las variables del estudio y sus dimensiones.

La comprensión lectora fue medida con la sección de razonamiento verbal del libro de ejercicios de la PAEP. Esta prueba consta de 24 ítems, otorgando un punto por cada respuesta correcta, cuyo puntaje se presenta en la Tabla 2.

La primera dimensión de estrategias globales de lectura cuenta con 13 ítems, la segunda de estrategias de resolución de problemas consta de ocho ítems y la tercera dimensión de estrategias de apoyo a la lectura cuenta con nueve ítems.

El rango de valores observado para cada dimensión, así como la media y desviación típica, se presentan en la Tabla 2.

\section{Tabla 2}

Estadísticos descriptivos de las variables del estudio

\begin{tabular}{lccccc}
\hline & \multicolumn{2}{c}{ Rango observado } & & \\
\cline { 2 - 3 } \multicolumn{1}{c}{ Variable } & Mínimo & Máximo & & $M$ & DE \\
\hline Comprensión lectora & 1 & 16 & 8.37 & 3.49 \\
Estrategias globales & 2.31 & 5.00 & 3.59 & 0.61 \\
Estrategias de apoyo & 1.89 & 5.00 & 3.29 & 0.70 \\
Estrategias de resolución de problemas & 1.63 & 5.00 & & 3.76 & 0.70 \\
\hline
\end{tabular}

\section{Análisis de predicción}

A fin de comprobar si las estrategias metacognitivas de lectura eran predictoras significativas de la comprensión lectora en los estudiantes se recurrió al análisis de regresión lineal múltiple. Los resultados revelaron que la puntuación general de las estrategias metacognitivas de lectura predicen significativamente la comprensión lectora $(F(3,86)=7.590, p$ $=.000, R=.458, R^{2}=.209, R^{2}$ corregida

\section{$=.182)$.}

Los coeficientes beta tipificados de las dimensiones de la variable estrategias metacognitivas de lectura asumieron los siguientes valores: estrategias globales, igual a -.008; estrategias de apoyo, igual a -.600; y estrategias de resolución de problemas, igual a .280. Esto demuestra que la dimensión estrategias globales de lectura es la que menos aporta a la comprensión lectora. 
Los datos precedentes muestran que el mejor modelo predictor está formado por las estrategias de apoyo y las de resolución de problemas $(F(2,87)=$ $11.516, p=.000, R=.458, R^{2}=.209, R^{2}$ corregida $=.191)$. Los coeficientes beta estandarizados para este mejor modelo predictor son iguales a -.602 y .275 , para los aportes de las estrategias de apoyo y para las de resolución de problemas, respectivamente.

\section{Otros hallazgos}

Se realizaron otros análisis para determinar los efectos significativos de las variables demográficas género, facultad y edad.

Se encontraron diferencias significativas en los resultados de la regresión lineal múltiple entre los géneros. Se observaron diferencias significativas de nivel de predicción de la comprensión lectora a partir de las estrategias metacognitivas de lectura entre la población masculina y la femenina. Entre los hombres la predicción resultó significativa $(\mathrm{F}(3,47)=5.787, p=.002, \mathrm{R}=.519$, $R^{2}=.270, R^{2}$ corregida = .223), mientras que no ocurrió lo mismo con las mujeres $(F(3,35)=2.310, p=.093, R=$ $.407, R^{2}=.165, R^{2}$ corregida $\left.=.094\right) . \mathrm{Si}$ se toma el mejor modelo predictor, en ambas subpoblaciones constituido por las estrategias de apoyo, la magnitud de la predicción es notablemente mayor en los hombres $(F(1,49)=15.605, p=.000$, $R=.491, R^{2}=.242, R^{2}$ corregida $\left.=.226\right)$ que en las mujeres $(F(2,36)=3.482, p=$ $.041, R=.403, R^{2}=.162, R^{2}$ corregida $=.116$ ). Entre los hombres, el aporte de las estrategias de apoyo tiene una correlación negativa más alta $(\beta=-.491)$ que entre las mujeres $(\beta=-.322)$.

No se observaron efectos significativos de la edad ni de la facultad sobre la comprensión lectora.

\section{Discusión}

El propósito de esta investigación fue conocer la relación entre las variables comprensión lectora y uso de estrategias metacognitivas. Es importante señalar que existen pocos estudios realizados con ambas variables. No se encontraron estudios anteriores que utilicen ambos instrumentos en una misma investigación y con una población similar.

Entre los resultados obtenidos se descubrió que existe una relación significativa entre la comprensión lectora y el uso de estrategias metacognitivas de lectura, lo cual significa que cuando los alumnos hacen uso de estrategias en lectura los niveles de comprensión lectora pueden aumentar o disminuir. Este hallazgo coincide con los resultados de otros estudios (Magogwe, 2013; Nasab y Motlagh, 2015) que afirman que, cuando los estudiantes son expuestos a estrategias metacognitivas adecuadas de comprensión de textos, adquieren una alta capacidad de lectura.

Asimismo, concuerda con los hallazgos de Hernández Vasquez (2015), quien señala que el empleo de las estrategias metacognitivas favorece la comprensión de lectura, ya que presentan una relación altamente significativa.

Uno de los hallazgos más interesantes y desafiantes de esta investigación está relacionado con el aporte de las dimensiones de la variable estrategias metacognitivas de lectura sobre la comprensión lectora. Se encontró que la dimensión estrategias globales es la que menos aporta a la comprensión lectora. Altunkaya y Sülükçü (2018) coinciden en que tales estrategias de lectura no influyen en el nivel de comprensión de lectura. Por el contrario, Hernández Vasquez (2015) señala que, en su estudio, las estrategias metacognitivas más empleadas y con un 
mayor aporte a la comprensión fueron las estrategias globales. El débil aporte de las estrategias globales en el estudio aquí reportado puede atribuirse a que los alumnos no suelen usarlas, ya que se refieren al conocimiento previo, la idea general o la concentración que se debe emplear al leer un texto. En general, los alumnos no las utilizan porque las desconocen.

En el presente estudio las estrategias de apoyo son las que más aportan a la comprensión lectora, pero de manera negativa, lo cual significa que a mayor comprensión menor uso de estrategias de apoyo. Estos resultados contrastan en parte con los de Gómez López et al. (2014), donde la puntuación del nivel de comprensión lectora tuvo correlaciones significativas con las subescalas estrategias globales y resolución de problemas, mientras que las estrategias de apoyo no correlacionaron significativamente con el nivel de comprensión lectora.

Es importante subrayar que tales estrategias están relacionadas con el procesamiento macro-estructural de los textos y con la comprensión de las ideas principales, como el uso de materiales (diccionario y notas) y acciones (preguntas, parafraseo o subrayado).

La correlación negativa puede atribuirse a que se trata de estrategias menos utilizadas por los estudiantes, probablemente porque no las consideran relevantes, requieren de mayor tiempo de realización o no fueron enseñadas. Donne y Rugg (2015) y Altunkaya y Sülükçü (2018) coinciden en que los estudiantes con un nivel alto de comprensión lectora no suelen usar las estrategias de apoyo, ya que se concentran en la complejidad del texto y no consideran importante usar tales estrategias. Muchos estudian- tes se comportan como lectores pasivos $y$, por tanto, su conocimiento previo interacciona menos de lo esperado con el texto y con el contexto.

En cuanto a la dimensión estrategias de resolución de problemas, en el presente estudio se encontró que fueron las estrategias más usadas por los estudiantes. Por su parte, Sariçoban y Behjoo (2017) encontraron una relación general significativa entre el instrumento MARSI y las habilidades de lectura de los estudiantes en términos de logros, lo cual quiere decir que los más exitosos prefieren utilizar sus estrategias de resolución de problemas más que las de las otras dos dimensiones. La estrategia más utilizada en la presente investigación, perteneciente a dicha dimensión, fue la estrategia evaluada con el ítem "Intento volver atrás (en la lectura) cuando pierdo la información" $(M=4.07)$. Este último hallazgo coincide con los del estudio de Khoshsima y Samani (2015), quienes encontraron que esta estrategia fue la más utilizada por los alumnos $(M=$ 3.77) con un nivel alto de comprensión de textos.

Los estudiantes a menudo usan más las estrategias de resolución de problemas con textos de ciencia que con textos de estudios sociales, ya que los participantes perciben que los textos científicos son más difíciles de leer que los textos de estudios sociales. Es por eso que reportan que las estrategias con el mayor porcentaje de participantes fueron todas las estrategias de resolución de problemas (Donne y Rugg, 2015; Gómez López et al., 2014).

Por el contrario, el estudio de Hernández Vasquez (2015) reveló que la dimensión de estrategias metacognitivas menos utilizada corresponde a la resolución de problemas. 


\section{CHABLÉ TOLOSA}

Por su parte, Smith-Keita (2018) enfatiza la relación moderadamente positiva entre el conocimiento metacognitivo de las estrategias de lectura de los estudiantes con respecto al uso de las estrategias de lectura globales y su logro con los estándares de preparación universitaria y profesional. Los datos indicaron que la instrucción de estrategia metacognitiva tiene un impacto positivo en los estudiantes de recuperación de la universidad en el área de lectura (Lee, 2018). Es importante señalar que cuando los alumnos tienen un conocimiento de tales estrategias y saben su uso se eleva el nivel de comprensión lectora al aplicarlas.

Respecto del género, se encontró que los hombres obtuvieron una mejor correlación entre las variables estudiadas que las mujeres. Se trata de un resultado que coloca un nuevo desafío para una investigación próxima. Con todo, las estrategias más usadas por ambos géneros fueron las estrategias de resolución de problemas y las menos utilizadas fueron las estrategias de apoyo, lo cual concuerda con Altunkaya y Sülükçü (2018) y Sariçoban y Behjoo (2017). Se puede inferir que los alumnos prefieren tales estrategias para aumentar su comprensión o cuando piensan que el texto es difícil y complicado. Por el contrario, Donne y Rugg (2015) no encontraron diferencias significativas en el uso de la estrategia sobre la comprensión lectora, en cuanto a género, grado matriculado o edad.

Se considera importante realizar estudios adicionales para determinar si con los mismos instrumentos y con estudiantes universitarios de otras escuelas y de diferentes niveles de avance en sus programas, los resultados siguen siendo similares.

\section{Referencias}

Ahmadi, M. R., Ismail, H. N. y Abdullah, M. K. K. (2013). The importance of metacognitive reading strategy awareness in reading comprehension. English Language Teaching, 6(10), 235-244. https:// doi.org/10.5539/elt.v6n10p235

Alaís Grillo, A., Leguizamón Sotto, D. V. y Sarmiento Ceballos, J. I. (2015). Comprensión lectora y desarrollo de estrategias cognitivas con el apoyo de un recurso TIC. Educación y Desarrollo Social, 9(1), 132-143. https://doi.org/10.18359/reds.556

Altunkaya, H. y Sülükçü, Y. (2018). The correlation between reading strategy metacognitive awareness levels and reading comprehension skills of 7th grade students. Uluslararası Türkçe Edebiyat Kültür Eğitim Dergisi, 7(4), 2502-2517. http://dergipark.gov.tr/teke/issue/42447/511191

Aşıkcan, M. y Saban, A. (2018). Prospective teacher's metacognitive awareness levels of reading strategies. Cypriot Journal of Educational Science, 8(1), 23-30. https://doi.org/10.18844/cjes. v13i1.3366

Basaraba, D., Yovanoff, P., Alonzo, J. y Tindal, G. (2013). Examining the structure of reading comprehension: Do literal, inferential, and evaluative comprehension truly exist? Reading and Writing: An Interdisciplinary Journal, 26(3), 349-379. https://doi.org/10.1007/s11145-0129372-9

Beltrán Campos, S. y Repetto Talavera, E. (2006). El entrenamiento en estrategias sobre la comprensión lectora del enunciado del problema aritmético: un estudio empírico con estudiantes de educación primaria. Revista Española de Orientación y Psicopedagogía, 17(1), 33-48. https://doi.org/10.5944/ reop.vol.17.num.1.2006.11336

Channa, M. A., Nordin, Z. S., Siming, I. A., Chandio, A. A. y Koondher, M. A. (2015). Developing reading comprehension through metacognitive strategies: A review of previous studies. English Language Teaching, 8(8), 181-186. https://doi.org/10.5539/elt.v8n8p181 
Dabarera, C., Renandya, W. A. y Zhang, L. J. (2014). The impact of metacognitive scaffolding and monitoring on reading comprehension. System, 42, 462-473. https://doi.org/10.1016/j. system.2013.12.020

Díaz Pertuz, E. M. y Quiroz Mosquera, J. L. (2016). Desarrollo de la comprensión lectora mediante el apoyo de ambientes virtuales de aprendizaje [Tesis de maestría, Universidad del Norte]. Repositorio Institucional de la Universidad del Norte. http://manglar.uninorte.edu.co/handle/10584/7520\#page=1

Donne, V. y Rugg, N. (2015). Comprehension practices of students who are deaf and hard of hearing. The Volta Review, 115(2), 101-127. https://doi.org/10.17955/tvr.115.2.760

Gómez López, A., Solaz Portolés, J. J. y Sanjosé López, V. (2014). Competencia en lengua inglesa de estudiantes universitarios españoles en el contexto del EEES: nivel de dominio lingüístico, estrategias metacognitivas y hábitos lectores. Revista de Educación, (363), 154-183. https://doi.org/104438/1988-592X-RE-2012-363-175

Gómez López, L. F. y Silas Casillas, J. C. (2012). Impacto de un programa de comprensión lectora. Revista Latinoamericana de Estudios Educativos, 42(3), 35-63. https://www.cee.edu.mx/rlee/revista/ r2011-2020/r_texto/t_2012_3_03.pdf

Gordillo Alfonso, A. y Flórez, M. P. (2009). Los niveles de comprensión lectora: hacia una enunciación investigativa y reflexiva para mejorar la comprensión lectora en estudiantes universitarios. Actualidades Pedagógicas, 53, 95-107. https://doi.org/10.19052/ap.1048

Green, L. B. y Roth, K. L. (2013). Increasing inferential reading comprehension skills: A single case treatment study. Canadian Journal of Speech-Language Pathology and Audiology, 37(3), 228-239. https:// www.cjslpa.ca/download.php?file=/2013_CJSLPA_Vol_37/No_03_182-245/Green_Roth_Fall_2013.pdf

Habibian, M. (2015). The impact of training metacognitive strategies on reading comprehension among ESL learner's. Journal of Education and Practice, 6(28), 61-69. https://www.iiste.org/Journals/index. $\mathrm{php} / \mathrm{JEP} /$ article/view/26555

Hernández Vasquez, N. E. (2015). Estrategias metacognitivas y comprensión de lectura en los estudiantes de Psicología de una Universidad Nacional [Tesis de maestría, Universidad César Vallejo]. Repositorio de la Universidad César Vallejo. http://repositorio.ucv.edu.pe/handle/20.500.12692/7447

Instituto Nacional para la Evaluación de la Educación. (2016). México en PISA 2015. https://www.inee. edu.mx/publicaciones/mexico-en-pisa-2015

Instituto Tecnológico y de Estudios Superiores de Monterrey. (2003). Guía de ejercicios de la Prueba de Admisión a Estudios de Posgrado. http://sitios.itesm.mx/va/calidadacademica/files/InstructivoPAEP.pdf

Jou, Y. J. (2015). Investigation of technological university students' use of metacognitive reading strategies in first and second languages. English Language Teaching, 8(1), 180-188. https://doi. org/10.5539/elt.v8n1p180

Khoshsima, H. y Samani, E. A. (2015). The most frequent metacognitive strategies used in reading comprehension among ESP learners. Advances in Language and Literary Studies, 6(1), 180-188. https://doi.org/10.7575/aiac.alls.v.6n.1p.180

Lee, J. A. (2018). The impact of metacognitive strategies taught in a college level developmental education class on comprehension, metacognitive awareness and self-efficacy (Publicación $\mathrm{N}^{\circ}$ 10933362) [Tesis doctoral, University of St. Francis]. ProQuest Dissertations and Theses Global.

Magogwe, J. M. (2013). Metacognitive awareness of reading strategies of University of Botswana English as second language students of different academic reading proficiencies. Reading and Writing, 4(1), 1-8. https://doi.org/10.4102/rw.v4i1.29

Muñoz-Muñoz, Á. E. y Ocaña de Castro, M. (2017). Uso de estrategias metacognitivas para la comprensión textual. Cuadernos de Lingüística Hispánica, 29, 223-244. https://doi. org/10.19053/0121053X.n29.2017.5865 


\section{CHABLÉ TOLOSA}

Nasab, M. S. B. y Motlagh, S. F. P. (2015). A complete review for metacognitive, cognitive, and social/ affective strategies as essential components of learning strategies and their relationships with EFL learners' reading comprehension promotion. Advances in Language and Literary Studies, 6(3), 166184. https://doi.org/10.7575/aiac.alls.v.6n.3p.166

Omar, S. y Bidin, A. (2015). The impact of multimedia graphic and text with autistic learners in reading. Universal Journal of Educational Research, 3(12), 989-996. https://doi.org/10.13189/ ujer.2015.031206

Oseda Gago, D. y Cabezudo Ríos, M. L. (2014). Comprensión lectora y resolución de problemas en estudiantes de educación primaria bilingüe en comunidades shipibas. Apuntes de Ciencia y Sociedad, 4(2), 185-195. https://doi.org/10.18259/acs.2014021

Salas Navarro, P. (2012). El desarrollo de la comprensión lectora en los estudiantes del tercer semestre del nivel medio superior de la Universidad Autónoma de Nuevo León [Tesis doctoral, Universidad Autónoma de Nuevo León]. Colección Digital UANL. http://cd.dgb.uanl.mx/handle/201504211/5454

Sánchez Carlessi, H. (2013). La comprensión lectora, base del desarrollo del pensamiento crítico: segunda parte. Horizonte de la Ciencia, 3(5), 31-38. http://revistas.uncp.edu.pe/index.php/ horizontedelaciencia/article/view/192

Sariçoban, A. y Behjoo, B. M. (2017). Metacognitive awareness of Turkish EFL learners on reading strategies. Journal of Graduate School of Social Sciences, 21(1), 159-172. http://dergipark.gov.tr/ ataunisosbil/issue/35347/425775

Schmitt, M. C. y Baumann, J. F. (1990). Metacomprehension during basal reading instruction: Do teachers promote it? Reading Research and Instruction, 29(3), 1-13. https://doi.org/10.1080/ 19388079009558012

Smith-Keita, D. R. (2018). The relationship between metacognitive awareness of reading strategies use and 10th grade students' college and career readiness achievement in English language arts (Publication $\mathrm{N}^{\circ}$ 10793385) [Tesis doctoral, The University of Mississippi]. ProQuest Dissertations and Theses Global.

Vallejos Atalaya, M. (2012). La motivación, la actitud hacia las ciencias, la ansiedad y las estrategias metacognitivas de lectura en el rendimiento de los estudiantes universitarios: un análisis longitudinal [Tesis doctoral, Universidad Complutense de Madrid]. Repositorio abierto de la UCM. https://eprints. ucm.es/17020/

Recibido: 21 de mayo de 2020

Revisado: 6 de junio de 2020

Aceptado: 6 de julio de 2020 